\title{
Les tests de langues
}

Christine Tagliante, Bruno Mègre, Gilles Breton, Dorothée Dupleix et Catherine Houssa

\section{OpenEdition}

Journals

Édition électronique

URL : https://journals.openedition.org/ries/1474

DOI : $10.4000 /$ ries. 1474

ISSN : 2261-4265

\section{Éditeur}

France Education international

\section{Édition imprimée}

Date de publication : 1 décembre 2004

Pagination : 115-121

ISBN : 978-2-85420-564-0

ISSN : $1254-4590$

\section{Référence électronique}

Christine Tagliante, Bruno Mègre, Gilles Breton, Dorothée Dupleix et Catherine Houssa, « Les tests de langues », Revue internationale d'éducation de Sèvres [En ligne], 37 | décembre 2004, mis en ligne le 18 novembre 2011, consulté le 06 juillet 2021. URL : http://journals.openedition.org/ries/1474 ; DOI : https://doi.org/10.4000/ries.1474

Ce document a été généré automatiquement le 6 juillet 2021.

(c) Tous droits réservés 


\title{
Les tests de langues
}

\author{
Christine Tagliante, Bruno Mègre, Gilles Breton, Dorothée Dupleix et \\ Catherine Houssa
}

1 Les politiques linguistiques menées par la Commission européenne et le Conseil de l'Europe ont ouvert la voie à des innovations profondes dans les pratiques de l'évaluation des compétences en langues. Ces deux grandes institutions, auxquelles il est nécessaire d'ajouter l'association ALTE ${ }^{1}$, disposent d'experts dont le travail consiste à donner vie, à travers le linguistique, aux objectifs éducatifs qu'elles se sont fixées.

Depuis la publication, en 2001, du Cadre européen commun de référence pour les langues ${ }^{2}$, dont les sous-titres sont "apprendre, enseigner, évaluer", de nombreuses améliorations ont vu le jour dans les pratiques d'évaluation des degrés de maîtrise des langues.

Le Cadre, diffusé et traduit en dix-huit langues ${ }^{3}$, présente une échelle de six niveaux de compétences en langues à laquelle les concepteurs de programmes, les auteurs de manuels d'enseignement et les évaluateurs peuvent se référer. Cette échelle est liée à un référentiel qui, sans prétendre être normatif ou universel, permet l'élaboration de programmes ou de certifications comparables. À ce titre, le Cadre constitue un instrument exemplaire pour la comparaison internationale des résultats de l'évaluation en langues. Il résout grandement le problème des équivalences de diplômes, les compétences et les niveaux étant rapportables à la même échelle de compétences, et cela pour toutes les langues.

\section{De nouveaux défis}

4 L'accroissement de la mobilité des personnes entraîne une augmentation de la demande d'apprentissage en langues et, partant, de la nécessité de certifications fiables et officielles en langues. Afin de faire face à ce défi, et devant la diversité des diplômes, tests et examens de langues émanant d'organismes non spécialisés, les autorités éducatives ont été amenées à mettre en place des outils permettant le transfert des qualifications. À cet effet, le Conseil de l'Europe propose un manuel ${ }^{4}$ qui permet de relier tous les examens de langues aux six niveaux qu'il a définis. 
5 Les organismes certificateurs qui ont intégré ces nouvelles données ont gagné en qualité et en rigueur, tant en ce qui concerne les procédures d'élaboration des évaluations que leurs contenus.

\section{Une grande rigueur dans les processus d'élaboration}

6 L'évaluation en langue est devenue un marché économique important ${ }^{5}$. De nombreux organismes et institutions élaborent aujourd'hui des tests de langue. Ces tests sont payants. La vitesse à laquelle ils se développent montre l'importance que le public leur donne.

7 Le mot "test», apparu en 1895, désigne une épreuve de mesure, de vérification. Pichot ${ }^{6}$ le définit ainsi: "Situation standardisée servant de stimulus à un comportement qui est évalué par la comparaison avec celui d'individus placés dans la même situation, afin de classer le sujet, soit quantitativement, soit typologiquement. » Gilbert de Landsheere ${ }^{7}$ ajoute que " pour mériter le nom de test, un examen doit être standardisé, fidèle, valide et étalonné ».

8 La standardisation vise à rendre les conditions de passation comparables d'une session à une autre et porte sur le matériel du test, la situation de passation, les consignes et les modalités de correction. L'étalonnage fait d'un test un instrument de mesure qui permet de situer un individu par rapport aux autres.

9 La sensibilité, ou finesse discriminative, est une qualité du test qui renvoie à son pouvoir classant : mettre en évidence des différences, même faibles, entre les individus. Par la suite, il est nécessaire de s'assurer que les différences observées sont stables : c'est ce qu'on nomme la « fidélité » du test. On ne s'intéresse à la troisième qualité, la validité, que si le test est fidèle : un test ne peut pas être valide s'il n'est pas fidèle. La validité est la qualité qui fait qu'un test mesure bien ce qu'il est censé mesurer.

\section{Les organismes certificateurs}

En tant que membres du groupe ALTE, les organismes producteurs ${ }^{8}$ des tests décrits ciaprès adhèrent à une charte de qualité dont l'objectif est de respecter les standards formels d'élaboration de tests de langue. Les membres de ALTE, en adoptant le Code de pratique de cette association, se sont engagés à expliciter ces standards, de façon à faire connaître et reconnaître la qualité de leurs tests, tant auprès des producteurs d'examens que des utilisateurs.

11 Seul l'ETS (Educational testing service), producteur américain du TOEFL, n'appartient pas au groupe ALTE. Les procédures d'élaboration de ce test répondent cependant aux standards internationaux.

\section{Des contenus communicatifs}

12 L'évaluation des langues n'est plus dominée par l'évaluation des structures linguistiques placées hors de tout contexte. Depuis la publication du Niveau Seuil (1975 pour l'anglais, 1976 pour le français), on évalue la capacité des individus à être autonomes dans leur pratique de la langue. Cette autonomie doit leur permettre de lier des relations avec les personnes dont ils apprennent la langue: échanger des 
informations, donner son opinion, raconter ses expériences, convaincre, argumenter, nuancer sa pensée.

13 Le Cadre européen commun de référence, adoptant les recherches des linguistes Canale et Swain (1981) ainsi que celles de Bachman (1990), propose un modèle actionnel. Il permet d'élaborer des évaluations qui traitent de la compétence langagière générale (interaction, production, réception, médiation), dans des domaines précis (personnel, public, éducationnel, professionnel), en réalisant des tâches communicatives dans des situations caractérisées par des lieux, des organismes, des acteurs, des objets, des événements.

\section{Vers une transparence des qualifications}

14 Des quatre tests analysés ci-après, trois ont été conçus dans le respect absolu de l'échelle de compétence du Cadre européen commun.

15 Le test d'allemand langue étrangère, le TestDaf, évalue trois niveaux de l'échelle commune (B1, B2, C1).

16 L'IELTS place les candidats sur une échelle de 1 à 9 , avec des correspondances vers l'échelle de référence.

17 Le test français TCF a été conçu dès le départ en référence à cette même échelle.

\section{Les perspectives}

On le voit, l'évaluation en langues se dirige vers une grande technicité, seule garante de la comparabilité des qualifications.

19 Mettre cette technicité au service des individus permet de leur fournir des certifications fiables et de s'assurer qu'ils disposent d'une réelle capacité de communication et d'échanges.

Dans les cursus scolaires actuels, il n'existe, sauf exception ${ }^{9}$, aucune validation à reconnaissance internationale du niveau de compétence en langues. L'épreuve de langue du diplôme de fin d'études ne met aucunement en évidence les expériences linguistiques. Bien souvent, cette épreuve évalue essentiellement la maîtrise de compétences passives. L'Europe disposant désormais de standards linguistiques, il est à souhaiter que l'avenir des tests de langues, de même que la validation des acquis, prenne place au sein des systèmes éducatifs et des cursus scolaires. Permettre aux élèves de quitter le système scolaire munis d'une certification monnayable sur le marché du travail serait la réponse de la technicité à l'un des défis posés par la mobilité.

\section{Christine Tagliante}




\section{Les tests allemand, anglais et français exigés pour l'entrée en université}

\section{Le TOEFL : Test of English as a foreign language}

21 Le TOEFL a été conçu et commercialisé depuis plus de cinquante ans par ETS, une entreprise appartenant au groupe américain Chauncey. Présent dans plus de cent quatre-vingts pays, le TOEFL s'est imposé auprès d'organismes gouvernementaux, d'entreprises et d'établissements scolaires et universitaires.

Le TOEFL est exigé comme preuve de compétence suffisante en anglais des étudiants étrangers ressortissants de pays non anglophones.

Le TOEFL existe en deux versions, une version informatisée et une version papier. Il se présente sous la forme d'un QCM (questionnaire à choix multiples). La durée de passation est différente d'une version à l'autre : trois heures trente pour la version papier, quatre heures pour la version informatisée (trente minutes d'instructions pour l'utilisation de l'ordinateur).

24 Le TOEFL assisté par ordinateur (TOEFL Computer Based Test) comporte quatre sections obligatoires. Il n'évalue pas l'expression orale.

25 Compréhension orale : capacité à comprendre les idées principales et secondaires de documents en anglais d'Amérique du Nord. Les questions sont orales et écrites. Trente à quarante-neuf questions, durée entre quarante et soixante minutes.

Structures : reconnaître les structures correctes d'un anglais écrit standard. Vingt à vingt-cinq questions, durée entre quinze et vingt minutes.

27 Compréhension écrite : comprendre de courts documents, dans différents niveaux de langues, dont ceux rencontrés dans les universités américaines. Quarante-quatre à cinquante-cinq questions, durée entre soixante-dix et quatre-vingt-dix minutes.

Expression écrite : rédiger en anglais à partir d'un sujet imposé tiré au sort. Trente minutes.

Le TOEFL sur version papier ne comporte que trois sections : compréhension orale; structures et expression écrite ; compréhension écrite.

30 Le TOEFL version papier peut être accompagné d'un test d'expression écrite : le Test of Written English (TWE). Le TWE, d'une durée de trente minutes, sert à compléter les résultats au TOEFL pour les candidats désirant poursuivre leurs études dans une université américaine, voire canadienne. Il évalue la capacité à organiser ses idées, argumenter, utiliser un anglais écrit standard.

\section{Les niveaux et les résultats}

31 Pour une inscription dans une université américaine, le niveau requis au TOEFL CBT est de 213 points sur de 300, score augmenté à 250 points dans certaines universités prestigieuses. Pour la version papier, le score minimum exigé est de 550 points sur un total de 677. Aucune indication de niveau n'est précisée, seul le score donne une information au candidat.

32 L'attestation du TOEFL est valable deux ans.

Bruno Mègre 


\section{L'IELTS : International English language testing service}

L'IELTS a été développé en 1989 et est géré conjointement par l'UCLES, le British Council et l'IDP Education Australia ${ }^{10}$. L'IELTS est reconnu par toutes les universités en Grande Bretagne, Australie et Nouvelle Zélande, la plupart des universités du Canada, de France ou d'Afrique du Sud ainsi que par de nombreuses universités européennes.

Il est reconnu dans le secteur professionnel par plus de mille deux cents institutions professionnelles ou gouvernementales.

Il existe trois cents centres répartis dans cent cinquante pays : quarante-huit dates de passation sont proposées dans l'année.

Il est destiné à toute personne ayant à étudier ou travailler dans un lieu où l'anglais est langue de communication et existe en deux formats: général (tout public) ou académique (inscription dans une université anglophone).

Les procédures d'élaboration de l'IELTS consistent en cinq grandes étapes : commande d'items, sélection, prétest, analyses des résultats pour la constitution de la banque d'items, publication du test à administrer.

Les quatre compétences linguistiques sont évaluées. Les épreuves de compréhension et d'expression écrites diffèrent selon le format choisi.

L'ordre des épreuves est toujours le suivant: compréhension orale : quarante items, quatre sections, trente minutes. Domaines: vie quotidienne et sociale, études ou formation. Types d'items: conversations ou monologues. Différents accents anglais sont utilisés. Types d'items: QCM, questions-réponses, phrases à compléter, classifications, appariements.

Compréhension écrite académique: quarante items, trois parties, soixante minutes. Textes extraits de magazines, livres ou journaux. Un des textes est obligatoirement argumentatif. Types d'items : QCM, appariement, titre à donner à un paragraphe.

41 Compréhension écrite générale : quarante items, trois parties, soixante minutes. Textes extraits de magazines, livres, journaux, publicités, modes d'emploi. Types de textes: factuels, descriptifs ou explicatifs, rarement argumentatifs. Même types d'items que pour le format académique.

42 Expression écrite académique : deux tâches, soixante minutes. Décrire et expliquer un tableau: organiser, présenter et comparer des données; décrire les étapes d'une procédure; expliquer le fonctionnement d'un système. Réagir à un point de vue: proposer une solution au problème; justifier une opinion; évaluer des idées ou des arguments.

43 Expression écrite générale : deux tâches, soixante minutes. Répondre à un problème donné en demandant des informations ou en expliquant une situation: écrire une correspondance personnelle; donner de l'information factuelle; exprimer des points de vue. Réagir à un point de vue : mêmes capacités que pour l'expression académique.

44 Expression orale: dix à quinze minutes: entretien entre deux candidats et un examinateur: réponses à des questions informatives sur la vie quotidienne du candidat; expression sur un thème proposé; entretien sur des questions plus abstraites. 


\section{Les niveaux de l'IELTS et les résultats} décrire communication typiques du contexte universitaire. Quatre parties, trente minutes.

\section{Les niveaux du TestDaF et les résultats}


correspondant environ à 700 heures de cours d'allemand avant de s'inscrire. Le candidat a la possibilité de se représenter à l'examen autant de fois qu'il le souhaite.

Les compétences sanctionnées correspondent à trois niveaux : TestDaF - niveau 5 (TDN 5), TestDaF - niveau 4 (TDN 4), TestDaF - niveau 3 (TDN 3).

Pour accéder à des études supérieures l'étudiant devra obtenir le TDN 5 . En deçà de ce niveau, le candidat pourra être admis à poursuivre des études supérieures sous condition de participation à des cours de langue allemande.

À un niveau inférieur au TDN 3, le TestDaF ne procède plus à une évaluation différenciée, mais constate simplement que le candidat n'a pas atteint le niveau minimum requis pour entreprendre des études supérieures dispensées en allemand.

Le résultat obtenu à chacune des quatre épreuves correspondantes se trouve mentionné séparément sur le certificat obtenu par le candidat. Le niveau global apparaît également.

\section{Dorothée Dupleix}

\section{Le TCF : Test de connaissance du français}

2 Le Centre international d'études pédagogiques (CIEP), répondant à la demande du ministère de l'Éducation nationale, de l'Enseignement supérieur et de la Recherche, a mis au point un outil d'évaluation rigoureux, simple et rapide d'utilisation, qui répond d'une part aux exigences des échanges internationaux et d'autre part à celles des établissements d'enseignement supérieur recevant des étudiants étrangers.

3 Le Test de connaissance du français (TCF) est aujourd'hui le test de référence en français langue générale pour les non francophones. Il a été officiellement lancé en janvier 2002 et a obtenu, dès janvier 2003, une certification ISO 9001, version 2000 pour la qualité de sa conception, son développement, sa gestion opérationnelle et commerciale. Les centres agréés, sous convention avec le CIEP, sont garants du respect de la norme de qualité.

64 En France, le TCF est présent dans cent quarante centres (universités, centres de langues, écoles d'architecture, Alliances françaises et grandes écoles (comme, par exemple, l'Institut des sciences politiques de Paris et l'Ecole polytechnique).

5 À l'étranger, cent vingt pays proposent le TCF dans plus de trois cent cinquante centres de passation.

Le TCF a deux types de public : un public généraliste (toute personne non francophone qui souhaite pour des raisons professionnelles ou personnelles, faire valider de façon simple et fiable ses connaissances du français); un public étudiant: le TCF est obligatoire pour toute inscription en premier cycle des établissements d'enseignement supérieurs français (arrêté du 21 novembre 2003). Souhaitant, comme les grands pays européens, se doter d'un test normé et transparent, le ministère a fait du TCF l'outil officiel de validation du niveau de français des candidats à la demande d'admission préalable en université (DAP).

Plus de cinquante mille candidats se sont déjà inscrits au test. Les candidats viennent de tous les continents et représentent cent cinquante-neuf pays différents.

Les épreuves : questionnaire à choix multiple de quatre-vingts items portant sur la compréhension orale, les structures de la langue et la compréhension écrite. Durée 
$1 \mathrm{~h}$ 30. Deux épreuves facultatives permettent d'évaluer en deux heures l'expression orale et écrite.

\section{Les niveaux, les résultats, la procédure d'élaboration} globalement et par compétences, sur l'un des six niveaux de l'échelle du Cadre européen commun. Le processus de production du test passe par les cinq grandes étapes classiques des test normés : commande d'items, sélection, prétests, analyses des résultats pour la constitution de la banque d'items, publication du test.

70 Tout au long des sessions et quelle que soit la version du test, les résultats restent fiables et comparables.

\section{Évolutions}

71

Le développement d'un TCF sur ordinateur est en projet en collaboration avec Cambridge-ESOL.

Catherine Houssa

\section{BIBLIOGRAPHIE}

À consulter

http://www.ets.org/toefl

http://www.ielts.org

TCF, Test de connaissance du français : http://www.ciep.fr

\section{NOTES}

1. ALTE : Association of language testers in Europe. Cette association regroupe trente organismes certificateurs européens, tous liés par une charte de qualité, producteurs d'examens et de tests dans vingt-quatre langues. http://www.alte.org

2. Cadre européen commun de référence pour les langues: apprendre, enseigner, évaluer, Division des politiques linguistiques - Conseil de l'Europe, Strasbourg - Didier, Paris 2001.

3. Allemand, anglais, basque, catalan, espagnol, finlandais, français, galicien, géorgien, hongrois, italien, japonais, polonais, portugais, moldave, russe, serbe et tchèque. D'autres traductions sont actuellement en cours.

4. Le Manuel pour relier les examens de langues au CECR est consultable sur : http://www.coe.int/T/ F/Coop\%E9ration\%5Fculturelle/education/Langues/Politiques_linguistiques/Manuel/ default.asp\#TopOfPage.

5. À titre indicatif, chaque année, plus d'un million de candidats passent les tests de l'université de Cambridge. Le TCF, lancé en janvier 2002, a déjà été présenté par plus de 50000 candidats.

Revue internationale d'éducation de Sèvres, 37 | décembre 2004 
6. Dictionnaire de l'évaluation et de la recherche en éducation, p. 295.

7. id.

8. Cambridge-ESOL pour le test IELTS ; les universités allemandes pour le test TestDaf ; le Centre international d'études pédagogiques (CIEP) pour le test TCF.

9. L'introduction des certifications internationales dans le système scolaire a été initiée par le ministère italien de l'Éducation en 2000. Cette tendance se développe aujourd'hui dans certains pays, en particulier en Europe.

10. International Development program of Australian universities and colleges.

INDEX

Mots-clés : test de langue, certification, langue allemande, langue anglaise, langue française

\section{AUTEURS}

\section{CHRISTINE TAGLIANTE}

Responsable du Pôle évaluation et certifications DELF DALF - TCF du Centre international d'études pédagogiques (CIEP).

\section{BRUNO MÈGRE}

Pôle évaluation et certifications DELF DALF - TCF du Centre international d'études pédagogiques (CIEP).

\section{GILLES BRETON}

Pôle évaluation et certifications DELF DALF - TCF du Centre international d'études pédagogiques (CIEP).

\section{DOROTHÉE DUPLEIX}

Pôle évaluation et certifications DELF DALF - TCF du Centre international d'études pédagogiques (CIEP).

\section{CATHERINE HOUSSA}

Pôle évaluation et certifications DELF DALF - TCF du Centre international d'études pédagogiques (CIEP). 